September 2, 1992

\title{
Deformation of the Wakimoto construction
}

\author{
A. Abada* ${ }^{*}$ A.H. Bougourzi ${ }^{\dagger}$ and M.A. El Gradechi ${ }^{\dagger}$ \\ * Institute for Theoretical Physics \\ State University of New York \\ Stony Brook, NY, 11794 USA \\ ${ }^{\dagger}$ CRM, Université de Montréal \\ C.P. 6128-A \\ Montréal, P.Q., Canada, H3C 3J7 \\ - Department of Mathematics \\ Concordia University \\ Montréal, P.Q., Canada, H4B 1R6
}

\begin{abstract}
We present the extension of the Wakimoto construction to the $s u(2)_{k}$ quantum current algebra and its associated $Z_{k}$ quantum parafermion algebra. This construction is achieved in terms of various deformations of three classical free boson fields. We also give the vertex operators corresponding to the quantum spin- $j$ representation.
\end{abstract}

\section{Introduction}

It is well established now that quantum groups and algebras play an important role in both twodimensional integrable models of quantum field theory and statistical mechanics, and conformal field theory [1, 2, 3, 4, 5, 6, 7, 8]. In particular, many authors have realized the importance of the infinite dimensional symmetries associated to quantum affine algebras (QAA) in constraining the integrable models. In fact, one hopes to carry over the success of the classical affine symmetries in chiral conformal field theory to the quantum case. In the classical case, it has been realized through many contributions that the Feigin-Fuchs construction (FFC) 9 is convenient for the technical resolution of conformal field theory, that is, it facilitates the study and the computation of several relevant physical quantities such as the correlation functions, the BRST-like cohomology structure, the irreducible characters and the fusion rules. This is because the FFC consists in representing the integrable models in terms of free fields whose properties are well known and simple. Therefore, the extension of the FFC to the quantum case and in particular to the QAA is highly desirable [6, 6, 10].

QAA were first introduced in Refs. [11, 12]. After that, the first FFC of the level one simply laced QAA was achieved by Frenkel and Jing [13]. Then the FFC of the spin-1/2 representation of the $s l(2)_{1}$ QAA followed in Ref. [14]. The FFC of the classical $s u(2)_{k}$ affine algebra was first obtained by Wakimoto [15] in terms of a pair of ghost fields and a free boson field. After that, other equivalent Wakimoto constructions in terms of three free boson fields were considered [16, 17, 18]. This then led to the FFC of the $Z_{k}$ parafermion theory [19, 20, 21, 22]. 
The purpose of this paper is to extend the Wakimoto construction to the QAA $s u(2)_{k}$. This paper is organized as follows. In section 2, we review the $s u(2)_{k}$ QAA in the context of conformal field theory. More specifically, we define the operator product expansions satisfied by the currents generating the $s u(2)_{k}$ QAA, which is henceforth referred to as the $s u(2)_{k}$ quantum current algebra (QCA). In section 3, we first review the Frenkel-Jing construction of the $s u(2)_{1}$ QCA, which requires different $q$-deformations of a single free boson field. Then we use it $\grave{a}$ la Zamolodchikov and Fateev 223 to obtain the Wakimoto construction of the $s u(2)_{k}$ QCA. In this case, various $q$-deformations of the three classical free boson fields are necessary. As a by-product, the currents generating the $Z_{k}$ quantum parafermion algebra are defined and their FFC is given. In section 4 , we derive the vertex (intertwining) operators corresponding to the spin- $j$ representations of the $s u(2)_{k}$ QCA and its associated $Z_{k}$ quantum parafermion algebra.

\section{$2 \quad$ The $s u(2)_{k}$ quantum current algebra}

The $s u(2)_{k}$ QAA is generated by the operators $\left\{E_{n}^{ \pm}, H_{n}, \gamma^{ \pm}, n \in \mathbf{Z}\right\}$ as follows:

$$
\begin{aligned}
& \gamma^{ \pm} \in \quad \text { the center of the algebra, } \\
& {\left[H_{n}, H_{m}\right]=\delta_{n+m, 0} \frac{[2 n]}{2 n} \frac{\gamma^{k n}-\gamma^{-k n}}{q-q^{-1}}, \quad n \neq 0,} \\
& {\left[H_{0}, H_{m}\right]=0,} \\
& {\left[H_{n}, E_{m}^{ \pm}\right]= \pm \sqrt{2} \frac{\gamma^{\mp|n| k / 2}[2 n]}{2 n} E_{n+m}^{ \pm}, \quad n \neq 0,} \\
& {\left[H_{0}, E_{m}^{ \pm}\right]= \pm \sqrt{2} E_{m}^{ \pm},} \\
& {\left[E_{n}^{+}, E_{m}^{-}\right]=\frac{\gamma^{k(n-m) / 2} \Psi_{n+m}-\gamma^{k(m-n) / 2} \Phi_{n+m}}{q-q^{-1}},} \\
& E_{n+1}^{ \pm} E_{m}^{ \pm}-q^{ \pm 2} E_{m}^{ \pm} E_{n+1}^{ \pm}=q^{ \pm 2} E_{n}^{ \pm} E_{m+1}^{ \pm}-E_{m+1}^{ \pm} E_{n}^{ \pm},
\end{aligned}
$$

where $\left.n, m \in \mathbf{Z},[n]=\left(q^{n}-q^{-n}\right) / q-q^{-1}\right), q$ is the deformation parameter, and $\Psi_{n}$ and $\Phi_{n}$ are respectively the modes of the fields $\Psi(z)$ and $\Phi(z)$ ( $z$ is a complex variable). These fields are defined by

$$
\begin{aligned}
& \Psi(z)=\sum_{n \geq 0} \Psi_{n} z^{-n}=q^{\sqrt{2} H_{0}} \exp \left[\sqrt{2}\left(q-q^{-1}\right) \sum_{n>0} H_{n} z^{-n}\right] \\
& \Phi(z)=\sum_{n \leq 0} \Phi_{n} z^{-n}=q^{-\sqrt{2} H_{0}} \exp \left[-\sqrt{2}\left(q-q^{-1}\right) \sum_{n<0} H_{n} z^{-n}\right] .
\end{aligned}
$$

Note that the algebra (1)-(7) coincides with the usual $s u(2)_{k}$ affine algebra [24] in the limit $q \rightarrow 1$.

In order to derive the extension of the Wakimoto construction to the above algebra it is convenient to express the relations (2)-(7) as a QCA. This is a standard procedure in the context of conformal field theory. This QCA is generated by the currents $E^{ \pm}(z)$ and $H(z)$ (with $\gamma$ being replaced by its eigenvalue $q[13,14)$, which are defined as the generating functions of $E_{n}^{ \pm}$and $H_{n}$ respectively, i.e.,

$$
\begin{aligned}
E^{ \pm}(z) & =\sum_{n=-\infty}^{+\infty} E_{n}^{ \pm} z^{-n-1}, \\
H(z) & =\sum_{n=-\infty}^{+\infty} H_{n} z^{-n-1} .
\end{aligned}
$$


The relations (2)-(7) are then equivalent to the following QCA, which reads as operator product expansions (OPE) among $E^{ \pm}(z)$ and $H(z)$ :

$$
\begin{aligned}
& H(z) H(w)=\sum_{n>0} \frac{[2 n][n k]}{2 n} z^{-n+1} w^{n+1}+\text { regular, } \\
& H(z) E^{ \pm}(w)= \pm \frac{\sqrt{2}}{z}\left[1+\sum_{n>0} \frac{q^{\mp n k / 2}[2 n]}{2 n} z^{-n} w^{n}\right] E^{ \pm}(w)+\text { regular } \\
& E^{ \pm}(w) H(z)=\mp \sqrt{2} \sum_{n>0} \frac{q^{\mp n / 2}[2 n]}{2 n} z^{n-1} w^{-n} E^{ \pm}(w)+\text { regular, } \\
& E^{+}(z) E^{-}(w)=\frac{1}{w\left(q-q^{-1}\right)}\left[\frac{\Psi\left(w q^{k / 2}\right)}{z-w q^{k}}-\frac{\Phi\left(w q^{-k / 2}\right)}{z-w q^{-k}}\right]+\text { regular, } \quad|w|<\left\{\left|z q^{k}\right|,\left|z q^{-k}\right|\right\} \\
& E^{-}(w) E^{+}(z)=\frac{1}{w\left(q-q^{-1}\right)}\left[\frac{\Psi\left(w q^{k / 2}\right)}{z-w q^{k}}-\frac{\Phi\left(w q^{-k / 2}\right)}{z-w q^{-k}}\right]+\text { regular, } \quad|z|<\left\{\left|w q^{k}\right|,\left|w q^{-k}\right|\right\} \\
& \left(z-w q^{ \pm 2}\right) E^{ \pm}(z) E^{ \pm}(w)=\left(z q^{ \pm 2}-w\right) E^{ \pm}(w) E^{ \pm}(z)
\end{aligned}
$$

where "regular" refers to terms that are non-singular in the limit $z, w \rightarrow 0$ in (12)-(14) and $z \rightarrow$ $w q^{ \pm k}$ in (15) and (16) respectively. Note that in (15) and (16) we have used the identification $\sum_{n \geq 0} z^{n} \equiv(1-z)^{-1}$ with $|z|<1$. This identification will also be understood in the subsequent treatment, especially when defining the contours of the Cauchy integrals.

\section{The Wakimoto construction of the $s u(2)_{k}$ QCA}

As our construction relies on that of Frenkel-Jing in the case of $s u(2)_{1}$ QCA [13], let us first review the latter in the language of conformal field theory. It is given by

$$
\begin{aligned}
H(z) & =i \partial \xi^{0}(z) \\
E^{ \pm}(z) & =\exp \left[ \pm i \sqrt{2} \xi^{ \pm}(z)\right] .
\end{aligned}
$$

Here, $\xi^{0}(z)$ and $\xi^{ \pm}(z)$ are deformed free bosonic fields that are the generating functions of the basic oscillators $\alpha_{n}, n \neq 0$, and the operators momentum $P$ and position $Q$, that is,

$$
\begin{aligned}
\xi^{0}(z) & =Q-i P \ln z+i \sum_{n \neq 0} \frac{\alpha_{n}}{n} z^{-n} \\
\xi^{ \pm}(z) & =Q-i P \ln z+i \sum_{n<0} \frac{q^{ \pm n / 2}}{[n]} \alpha_{n} z^{-n}+i \sum_{n>0} \frac{q^{\mp n / 2}}{[n]} \alpha_{n} z^{-n}
\end{aligned}
$$

with

$$
\begin{aligned}
{\left[\alpha_{n}, \alpha_{m}\right] } & =\delta_{n+m, 0} \frac{[2 n][n]}{2 n} \\
{[Q, P] } & =i
\end{aligned}
$$

According to the usual definition of the normal ordering of $\alpha_{n}, P$ and $Q$ as given in Ref. [25], the relations (20)-(23) lead to the following two-point correlation functions:

$$
\left\langle\xi^{ \pm}(z) \xi^{\mp}(w)\right\rangle=-\frac{1}{2} \ln (z-w q)\left(z-w q^{-1}\right),
$$




$$
\begin{aligned}
\left\langle\xi^{ \pm}(z) \xi^{ \pm}(w)\right\rangle & =-\frac{1}{2} \ln (z-w)\left(z-w q^{\mp 2}\right) \\
\left\langle\partial \xi^{0}(z) \xi^{ \pm}(w)\right\rangle & =-z^{-1}\left[1+\frac{1}{2\left(q-q^{-1}\right)} \ln \left(\frac{z-w q^{-2 \mp 1 / 2}}{z-w q^{2 \mp 1 / 2}}\right)\right] .
\end{aligned}
$$

It can readily be seen through (24)-(26) that the $s u(2)_{1}$ QCA (12)-(17) is indeed satisfied.

Let us now make use $\grave{a}$ la Zamolodchikov and Fateev [23] of the Frenkel-Jing construction of $s u(2)_{1}$ QCA given in (18) and (19) to extend the Wakimoto construction to the $s u(2)_{k}$ QCA (12)-(17).

The generalization of (18) so that $H(z)$ satisfies (12) reads

$$
H(z)=i \sqrt{k} \partial \xi^{0}(z)
$$

where now the deformed bosonic field $\xi^{0}(z)$ is defined by

$$
\xi^{0}(z)=Q-i P \ln z+i \sum_{n \neq 0} \frac{\alpha_{n}}{n} z^{-n}
$$

with

$$
\begin{aligned}
{\left[\alpha_{n}, \alpha_{m}\right] } & =\delta_{n+m, 0} \frac{[2 n][n k]}{2 n k} \\
{[Q, P] } & =i
\end{aligned}
$$

Furthermore, the natural generalization of $E^{ \pm}(z)$ so that (13) and (14) are satisfied reads

$$
E^{ \pm}(z)=\exp \left[ \pm i \sqrt{\frac{2}{k}} \xi^{ \pm}(z)\right]
$$

with the deformed free bosonic fields $\xi^{ \pm}(z)$ being

$$
\xi^{ \pm}(z)=Q-i P \ln z+i k \sum_{n<0} \frac{q^{ \pm n k / 2}}{[n k]} \alpha_{n} z^{-n}+i k \sum_{n>0} \frac{q^{\mp n k / 2}}{[n k]} \alpha_{n} z^{-n} .
$$

However, $E^{ \pm}(z)$ as given in (31) do not satisfy the relations (15)-(17) of the $s u(2)_{k}$ QCA. Instead, they have the OPE:

$$
\begin{aligned}
& E^{ \pm}(z) E^{\mp}(w)=\exp \left[\frac{2}{k}\left\langle\xi^{ \pm}(z) \xi^{\mp}(w)\right\rangle\right]: E^{ \pm}(z) E^{\mp}(w): \\
& E^{ \pm}(z) E^{ \pm}(w)=\exp \left[-\frac{2}{k}\left\langle\xi^{ \pm}(z) \xi^{ \pm}(w)\right\rangle\right]: E^{ \pm}(z) E^{ \pm}(w):
\end{aligned}
$$

where

$$
\begin{aligned}
\left\langle\xi^{ \pm}(z) \xi^{\mp}(w)\right\rangle & =-\ln z+\frac{k}{2} \sum_{n>0} \frac{[2 n]}{n[n k]} z^{-n} w^{n}, \\
\left\langle\xi^{ \pm}(z) \xi^{ \pm}(w)\right\rangle & =-\ln z+\frac{k}{2} \sum_{n>0} \frac{q^{\mp n k}[2 n]}{n[n k]} z^{-n} w^{n} .
\end{aligned}
$$

In (33) and (34), the symbol :: denotes the normal ordering with respect to the bosonic operators $\alpha_{n}, P$ and $Q$ [25]. Clearly, the currents $E^{ \pm}(z)$ as given in (31) must be further corrected by 
including additional deformed bosonic fields in order to satisfy (15)-(17). To this end, let $\varphi_{1}(z)$ and $\varphi_{2}(z)$ be two new deformed bosonic fields with the expansions

$$
\begin{aligned}
& \varphi_{1}(z)=Q_{1}-i P_{1} \ln z+i \sum_{n \neq 0} \frac{\beta_{n}}{n} z^{-n}, \\
& \varphi_{2}(z)=Q_{2}-i P_{2} \ln z+i \sum_{n \neq 0} \frac{\lambda_{n}}{n} z^{-n},
\end{aligned}
$$

and

$$
\begin{aligned}
& {\left[\beta_{n}, \beta_{m}\right]=n \delta_{n+m, 0} I(n),} \\
& {\left[\lambda_{n}, \lambda_{m}\right]=-n \delta_{n+m} J(n),} \\
& {\left[Q_{1}, P_{1}\right]=i,} \\
& {\left[Q_{2}, P_{2}\right]=-i,}
\end{aligned}
$$

where $I(n)$ and $J(n)$ are given by

$$
\begin{aligned}
I(n) & =\frac{k^{2}[n][n(k+2) / 2]}{(k+2)[n k][n k / 2]}, \\
J(n) & =\frac{k}{4} \frac{[n(k+2)]+[2 n]-[n k]}{[n k]} .
\end{aligned}
$$

Note that all the remaining commutation relations are trivial, $\varphi_{2}(z)$ has a "time-like" signature, and in the limit $q \rightarrow 1, I(n), J(n) \rightarrow 1$ as expected. The currents $E^{ \pm}(z)$ that fully satisfy the $s u(2)_{k} \mathrm{QCA}(12)$ through (17) read then as follows:

$$
E^{ \pm}(z)=\frac{\exp \left[ \pm i \sqrt{\frac{2}{k}} \xi^{ \pm}(z)\right]}{z\left(q-q^{-1}\right)}\left\{\exp \left[i X^{ \pm}(z, q)\right]-\exp \left[i X^{ \pm}\left(z, q^{-1}\right)\right]\right\},
$$

where

$$
X^{ \pm}(z, q)= \pm \sqrt{\frac{2}{k}} \varphi_{2}\left(z q^{k / 2}\right)+\sqrt{\frac{k+2}{2 k^{2}}}\left[\varphi_{1}\left(z q^{k}\right)-\varphi_{1}(z)\right] .
$$

It can easily be checked that in the limit $q \rightarrow 1$ the correct classical expressions of the currents $E^{ \pm}(z)$ are recovered, namely, [18]

$$
E^{ \pm}(z)=i \sqrt{k}\left[ \pm \frac{\partial \varphi_{2}(z)}{\sqrt{2}}+\sqrt{\frac{k+2}{2 k}} \partial \varphi_{1}(z)\right] \exp \left\{ \pm i \sqrt{\frac{2}{k}}\left[\varphi_{2}(z)+\xi(z)\right]\right\},
$$

where $\xi(z)$ is the same classical limit of both $\xi^{ \pm}(z)$. For illustration, let us show that $E^{ \pm}(z)$ satisfy the relations (15) and (16). They have the OPE

$$
\begin{array}{ll}
E^{+}(z) E^{-}(w)=\Theta(z, w, q)+\text { regular, } & |w|<\left\{\left|z q^{k}\right|,\left|z q^{-k}\right|\right\}, \\
E^{-}(w) E^{+}(z)=\Theta(z, w, q)+\text { regular, } & |z|<\left\{\left|w q^{k}\right|,\left|w q^{-k}\right|\right\},
\end{array}
$$

with "regular" referring again to non-singular terms in the limit $z \rightarrow w q^{ \pm k}$, and $\Theta(z, w, q)$ being defined by

$$
\begin{aligned}
\Theta(z, w, q)= & -\frac{: \exp \left\{i \sqrt{\frac{2}{k}}\left[\xi^{+}(z)-\xi^{-}(w)\right]\right\}:\left\{\frac{q\left(z-w q^{-k-2}\right)}{z w\left(q-q^{-1}\right)^{2}}: \exp \left[i X^{+}(z, q)-i X^{-}\left(w, q^{-1}\right)\right]:\right.}{}\{ \\
& \left.+\frac{z-w q^{k+2}}{q\left(z-w q^{k}\right)}: \exp \left[i X^{+}\left(z, q^{-1}\right)-i X^{-}(w, q)\right]:\right\}
\end{aligned}
$$


Here the normal ordering is with respect to all the bosonic oscillators. Using now standard rules of deriving the commutation relations from the OPE [25], one finds that

$$
\left[E_{n}^{+}, E_{m}^{-}\right]=\frac{1}{(2 \pi i)^{2}} \oint_{C_{0}} d w w^{m} \oint_{C_{1}} d z z^{n} \Theta(z, w, q) .
$$

Because of the relations (48) and (49), the contours $C_{0}$ and $C_{1}$ enclose respectively the origin point $w=0$ and the poles $z=w q^{ \pm k}$. The contribution of the fields $\varphi_{1}(z)$ and $\varphi_{2}(z)$ cancel in a non-trivial way after performing the first integration in (51). In fact, only the contribution of the fields $\xi^{ \pm}(z)$ survives as expected, and because of the identities

$$
\begin{aligned}
& \Psi(z) \equiv: \exp \left\{i \sqrt{\frac{2}{k}}\left[\xi^{+}\left(z q^{k / 2}\right)-\xi^{-}\left(z q^{-k / 2}\right)\right]\right\}: \\
& \Phi(z) \equiv: \exp \left\{i \sqrt{\frac{2}{k}}\left[\xi^{+}\left(z q^{-k / 2}\right)-\xi^{-}\left(z q^{k / 2}\right)\right]\right\}:
\end{aligned}
$$

it leads to the correct relations (15) and (16) or equivalently to the relation (6). Finally, let us mention that the remaining relation (17) of the $s u(2)_{k}$ QCA is also satisfied.

Let us now introduce the "basic" $Z_{k}$ quantum parafermion currents referred to as $\psi_{1}(z)$ and $\psi_{1}^{\dagger}(z)$. As in the classical case, these currents are defined by 23.

$$
\begin{aligned}
& E^{+}(z)=\sqrt{k} \psi_{1}(z) \exp \left[i \sqrt{\frac{2}{k}} \xi^{+}(z)\right] \\
& E^{-}(z)=\sqrt{k} \psi_{1}^{\dagger}(z) \exp \left[-i \sqrt{\frac{2}{k}} \xi^{-}(z)\right] .
\end{aligned}
$$

The FFC of $\psi_{1}(z)$ and $\psi_{1}^{\dagger}(z)$ follows from that of $E^{ \pm}(z)$ as given in (45) and (46). It reads as follows:

$$
\begin{aligned}
\psi_{1}(z) & =\frac{\exp \left[i X^{+}(z, q)\right]-\exp \left[i X^{+}\left(z, q^{-1}\right)\right]}{\sqrt{k}\left(q-q^{-1}\right) z} \\
\psi_{1}^{\dagger}(z) & =\frac{\exp \left[i X^{-}(z, q)\right]-\exp \left[i X^{-}\left(z, q^{-1}\right)\right]}{\sqrt{k}\left(q-q^{-1}\right) z} .
\end{aligned}
$$

The FFC of the remaining currents $\psi_{\ell}(z)$ and $\psi_{\ell}^{\dagger}(z), \ell=2, \ldots, k$ generating the full quantum $Z_{k}$ parafermion algebra can be derived from that of $\psi_{1}(z)$ and $\psi_{1}^{\dagger}(z)$ through their OPE.

To recapitulate, the relations (27) and (45) describe the Wakimoto construction of the $s u(2)_{k}$ QCA (12)-(17) in terms of the deformed free bosonic fields $\xi^{0}(z), \xi^{ \pm}(z), \varphi_{1}(z)$ and $\varphi_{2}(z)$, which are given in (28), (32), (37) and (38) respectively. The FFC of the basic currents of the $Z_{k}$ quantum parafermion algebra, which are defined through (54) and (55), is given in (56) and (57).

\section{Vertex realization of the spin- $j$ representation}

The spin- $j$ representation of the $s u(2)_{k}$ QCA (12)-(14) is described by the " $q$-primary" fields $V^{j, m}(z) \equiv V^{m}(z)(m=-j, \ldots,+j)$ and their " $q$-descendants." $V^{j}(z)$, which creates the highest weight state from the bosonic vacuum, is such that

$$
\begin{aligned}
{\left[E^{+}(z), V^{j}(w)\right] } & =0 \\
{\left[H_{n}, V^{j}(w)\right] } & =j \sqrt{2}\left\{\delta_{n, 0}+\delta_{n>0} \gamma^{n k / 2} \frac{[n k]}{n k} w^{n}+\delta_{n<0} \gamma^{-n k / 2} \frac{[n k]}{n k} w^{n}\right\} V^{j}(w) .
\end{aligned}
$$


The FFC of $V^{j}(z)$ that is consistent with (58) and (59) is

$$
V^{j}(z)=\exp \left\{i j \sqrt{\frac{2}{k}}\left[\tilde{\xi}(z)+\tilde{\phi}_{2}(z)+\sqrt{\frac{k}{k+2}} \tilde{\phi}_{1}(z)\right]\right\}
$$

where

$$
\begin{aligned}
\tilde{\xi}(z) & =Q-i P \ln (-z)+2 i \sum_{n>0} \frac{q^{n k / 2}}{[2 n]} \alpha_{n} z^{-n}+2 i \sum_{n<0} \frac{q^{-n k / 2}}{[2 n]} \alpha_{n} z^{-n}, \\
\tilde{\phi}_{1}(z) & =Q_{1}-i P_{1} \ln (-z)+i \sum_{n>0} \frac{\beta_{n}}{n I(n)} z^{-n}+i \sum_{n<0} \frac{\beta_{n}}{n I(n)} z^{-n}, \\
\tilde{\phi}_{2}(z) & =Q_{2}-i P_{2} \ln (-z)+i \sum_{n>0} \frac{q^{n k / 2}+q^{-n k / 2}}{2 n J(n)} \lambda_{n} z^{-n}+i \sum_{n<0} \frac{q^{n k / 2}+q^{-n k / 2}}{2 n J(n)} \lambda_{n} z^{-n} .
\end{aligned}
$$

The FFC of the other fields $V^{m}(w)$ in the multiplet are obtained from that of $V^{j}(w)$ through the relation

$$
V^{m}(w)=\left[E_{0}^{-}, \ldots\left[E_{0}^{-}, V^{j}(w)\right]_{q^{k}} \ldots\right]_{q^{k}} .
$$

In (64) there are $j-m$ deformed commutators, which are defined by

$$
\left[E_{0}^{-}, V^{j}(w)\right]_{q^{k}} \equiv q^{k} E_{0}^{-} V^{j}(w)-V^{j}(w) E_{0}^{-} .
$$

Finally, let us note that the primary field $\chi^{j}(z)$ of the $Z_{k}$ quantum parafermion algebra is again defined as in the classical case, that is, through the relation

$$
V^{j}(z)=\chi^{j}(z) \exp \left[i j \sqrt{\frac{2}{k}} \tilde{\xi}(z)\right] .
$$

This means that the FFC of $\chi^{j}(z)$ is given by

$$
\chi^{j}(z)=\exp \left\{i j \sqrt{\frac{2}{k}}\left[\tilde{\phi}_{2}(z)+\sqrt{\frac{k}{k+2}} \tilde{\phi}_{1}(z)\right]\right\} .
$$

While typing this paper we received a preprint by A. Matsuo [26] discussing another deformation of the Wakimoto realization. However, he did not discuss the FFC of the spin- $j$ representation and the $Z_{k}$ quantum parafermion algebra introduced here.

\section{Acknowledgments}

This work was supported in part by funds provided by the Natural Sciences and Engineering Research Council (NSERC) of Canada, and the "Fonds FCAR pour la formation de chercheurs et l'aide à la recherche." We thank Professors Y. Saint-Aubin, V. Hussin and S.T. Ali for their encouragement.

\section{References}

[1] V. G. Drinfled, Proc. ICM (Am. Math. Soc., Berkeley, CA, 1986). 
[2] M. Jimbo, Commu. Math. Phys. 102, 537 (1986).

[3] S. L. Woronowicz, Comm. Math. Phys. 111, 613 (1987).

[4] L. Alvarez-Gaumé, C. Gomez and G. Sierra, Topics in conformal field theory, Knizhnik Memorial Volume (World Scientific, Singapore, 1990).

[5] H. J. De Vega, Int. J. Mod. Phys. A4, 2371 (1989).

[6] I. B. Frenkel and N. Yu Reshetikhin, Comm. Math. Phys. 146, 1 (1992).

[7] D. Bernard and A. Leclair, CLNS 92/1147, SPhT-92-054.

[8] B. Davies, M. Jimbo, T. Miwa and A. Nakayashiki, RIMS preprint, 873 (1992).

[9] B. L. Feigin and D. B. Fuchs, Funct. Anal. Appl. 16, 114 (1982); 17, 241 (1983).

[10] O. Babelon and D. Bernard, SPhT-92-062; LPTHE-92-20.

[11] V. G. Drinfeld, Soviet Math. Doklady 32, 254 (1985); 36, 212 (1988).

[12] M. Jimbo. Lett. Math. Phys. 10, 63 (1985).

[13] I. B. Frenkel and N. H. Jing, Proc. Nat'l. Acad. Sci. (USA) 85, 9373 (1988).

[14] M. Jimbo, K. Miki, T. Miwa and A. Nakayashiki, RIMS preprint, (1992).

[15] M. Wakimoto, Comm. Math. Phys. 104, 605 (1986).

[16] K. Ito and Y. Kazama, Mod. Phys. Lett. A5, 215 (1990).

[17] A. Gerasimov, A. Marshakov, A. Morozov, M. Olshanetsky and S. Shatashvili, Int. J. Mod. Phys. A5, 2495 (1990).

[18] A. H. Bougourzi, Phys. Lett. B286, 279 (1992).

[19] D. Nemeschansky, Phys. Lett. B224, 566 (1989) .

[20] T. Jayaraman, K. S. Narain and M. H. Sarmadi, Nucl. Phys. B343, 418 (1990).

[21] P. A. Griffin and O. F. Hernández, Int. J. Mod. Phys. A7, 1233 (1992).

[22] C. Ahn, S. Chung and S.-H. H. Tye, Nucl. Phys. B365, 191 (1991).

[23] A. B. Zamolodchikov and V. A. Fateev, Sov. Phys. J.E.T.P. 62, 215 (1985).

[24] V. G. Kac, Infinite dimensional Lie algebras, (Cambridge University Press, 1985).

[25] P. Goddard and D. Olive, Int. J. Mod. Phys. A1, 303 (1986).

[26] A. Matsuo, Nagoya University preprint, August 31, 1992. 